\title{
静电放电引发嵌入式软件故障的 等效实验预测方法
}

\author{
秦海潮 \\ ( 北京航空航天大学 电子信息工程学院, 北京 100191)
}

\begin{abstract}
摘 要: 对于电子设备的正常运行, 静电放电有时是一种严重威胁。目前, 由静电放电造成的大多数 硬件故障问题可以在产品设计阶段进行规避。但静电放电引发的嵌入式软件故障, 因其偶发性、突发 性、隐蔽性等特点, 依然是一项业界难题。将目前发现的嵌入式软件故障进行分类, 并提出了一系列 软件故障的等效实验监测方法。通过方法探究和大量实验佐证, 力图从这些监测方法中寻求静电放 电引发嵌入式软件故障的等效实验预测方法。
\end{abstract}

\section{关 键 词: 嵌入式软件测试; 静电放电; 实验设计; $\mathrm{WiFi}$ \\ 中图分类号: $\mathrm{V} 221^{+} .3$; TB553 \\ 文献标志码 : A \\ 文章编号 : 1000-2758(2018) 01-0195-08}

静电放电的过程能在数纳秒内产生数千伏的高 压和几十安培的电流, 并伴随强电磁场, 对电子设备 的正常运行是一种严重威胁 ${ }^{[1]}$ 。静电放电产生的 能量会通过辐射或传导的形式, 沿着最低阻抗路径 进行泄放。静电放电对电子设备造成的故障大致分 为 2 种 $^{[2-6]}$ :

a) 模块损坏。该类故障为过压、过流或强电磁 场引起的永久性故障;

b) 功能受扰。该类故障不对模块造成损坏, 为 临时性故障。这类故障又叫“软件故障” ${ }^{[6]}$ 。

当静电放电的等级低于使硬件造成损坏的等级 时, 嵌人式软件故障可能就会出现。这些软件故障 包括使电子设备关机、重启、应用软件故障或整个系 统软件故障 ${ }^{[7]}$ 。

由于在产品的研发过程中, 硬件的研发周期相 对更长, 而这类软件故障只有在搭载到硬件平台后 才可能被发现。而且, 由于静电放电引发的嵌人式 软件故障(下简称软件故障) 具有偶发性、突发性、 隐蔽性等特点, 少量的实验难以有效发现所有潜在 的软件故障。同样棘手的是, 在研发末期, 解决这类 问题更加耗时耗力, 而且措施十分有限。严重的软 件故障可能造成软件版本更新、硬件设计重来、产品 推迟发布等风险。
因此, 如果能在硬件平台尚未完全准备就绪之 前就能够发现这些软件故障, 这些问题将更容易解 决。换言之, 如果能够保证各模块不会因受静电放 电的影响而产生软件故障, 那么最终产品中出现类 似问题的概率将大幅降低。现在的问题是, 怎样在 研发阶段预测最终产品的软件故障。

2015 年, Charvaka 和 Harald 探讨了软件故障的 产生机理并给出了合理的设计建议 ${ }^{[3]}$ 。此后, Thomson 等人深人探究了 ESD 导致的系统级软件故 障,包括应用软件故障的发生场景 ${ }^{[4-5]}$ 、故障的复现 性 ${ }^{[6]}$ 和故障國值的评估方法 ${ }^{[7-8]}$ 。关于软件故障的 预测方法, Schwingshackl 等提出了全波仿真和暗室 内测试等预测方法 ${ }^{[7,9-13]}$ 。

本文应用了电压电流测量等常规方法, 同时创 新性地提出了将热成像分析法、快速短时傅里叶变 换分析和声音辨别法应用于软件故障的预测中。笔 者通过不同的实验方法, 试图找到对应软件故障的 指标变化和测试工具, 并通过对比设备各功能模块 运行状态下多种实验方法的测试结果, 从中选取备 选的软件故障预测方法。

在本文相关的测试中,使用 1 台传输线脉冲发 生器 ${ }^{[2]}$ (transmission line pulse generator, TLP) 来实 现静电放电的过程。TLP 发出的短脉冲通过接触放 
电、电阻耦合、二极管耦合 ${ }^{[4]}$ 或场耦合 ${ }^{[5]}$ 的方式对 受试品 (Device Under Test, DUT) 进行注人。笔者对 多型号的数码相机、等离子电视机、智能手机、笔记 本电脑和开发板等 DUT 进行了静电放电测试。根 据实验现象,发现 6 种不同类型的软件故障:

a) 软件失控。比如有触控功能的 DUT 在 TLP 注人后从一个前台应用切换到另一个前台应用。

b) 应用软件崩溃。实验中仅应用软件㿈疾, 系 统软件正常运行。

c) 系统软件崩溃。TLP 注人后整个系统软件 崩溃, 但系统供电正常。这类故障通常可以通过手 动复位解除。

d）显示错乱。对于一些带有显示屏的 DUT 来 说, 该类故障十分常见。比如, 显示屏会显示蓝色或 黑色条纹。

e) 系统掉电。TLP 注人后 DUT 掉电关机。

f) 其他。比如瞬时闭锁。

目前, 软件故障解决方案的主要瓶颈集中在 a 类和 $\mathrm{b}$ 类故障。将对以上各类故障举例示意, 以方 便读者深人了解。

\section{2 软件故障监测手段}

\section{1 监测直流电流法}

单个电源网络 ( power distribution network,
PDN) 中的电流变化理论上也可以作为一个监测指 标, 但因板上空间有限, 多个 PDN 相互靠近, 难有实 际准确的监测方案。因此, 可以用 DUT 供电线上的 电流变化作为部分 DUT 软件故障的监测指标。

本例将监测直流电流法应用于监测高通公司的 Dragon Board 开发板。该开发板使用 AM335x ARM Cortex-A8 CPU, 搭载 Linux 操作系统。TLP 充电电 压为 $2 \mathrm{kV}$, 使用小环天线进行注人。示波器型号为 R\&S RTO1024, 相关设置为: (1)使用 $1 \mathrm{M} \Omega$ 直流耦 合; (2)使用 $100 \mathrm{kHz}$ 低通滤波器; (3)采样频率设为 10 $\mathrm{MSa} / \mathrm{s}$; (4)触发方式为边沿触发, 使用 TLP 为外部触 发源; 5不使用差值,仅采集原始时域数据。

一个实验细节值得提及、由于注入环天线与 TLP 间阻抗不匹配, 可能在 TLP 和注人环天线间产 生反射。可以通过在环天线端串接 $50 \Omega$ 电阻来削 弱该反射。

实验配置如图 1 所示。对于直流电流监测而 言, 需要为所有参试设备提供一个公共参考板。因 此, 选择在 DUT 电源线的回线上增加一颗 $1 \Omega$ 的电 阻, 通过监测电阻两端的电压值监测供电线上的电 流值。为了降低电源适配器开关噪声潜在的影响, 可在电源线上并接电容。同时, 为了降低潜在的共 模噪声, 在示波器环天线引线上串接磁环。通过将 注人环天线停放在 DUT 不同的位置, 得到不同的软 件故障, 同时监测得到对应时刻的直流电流。

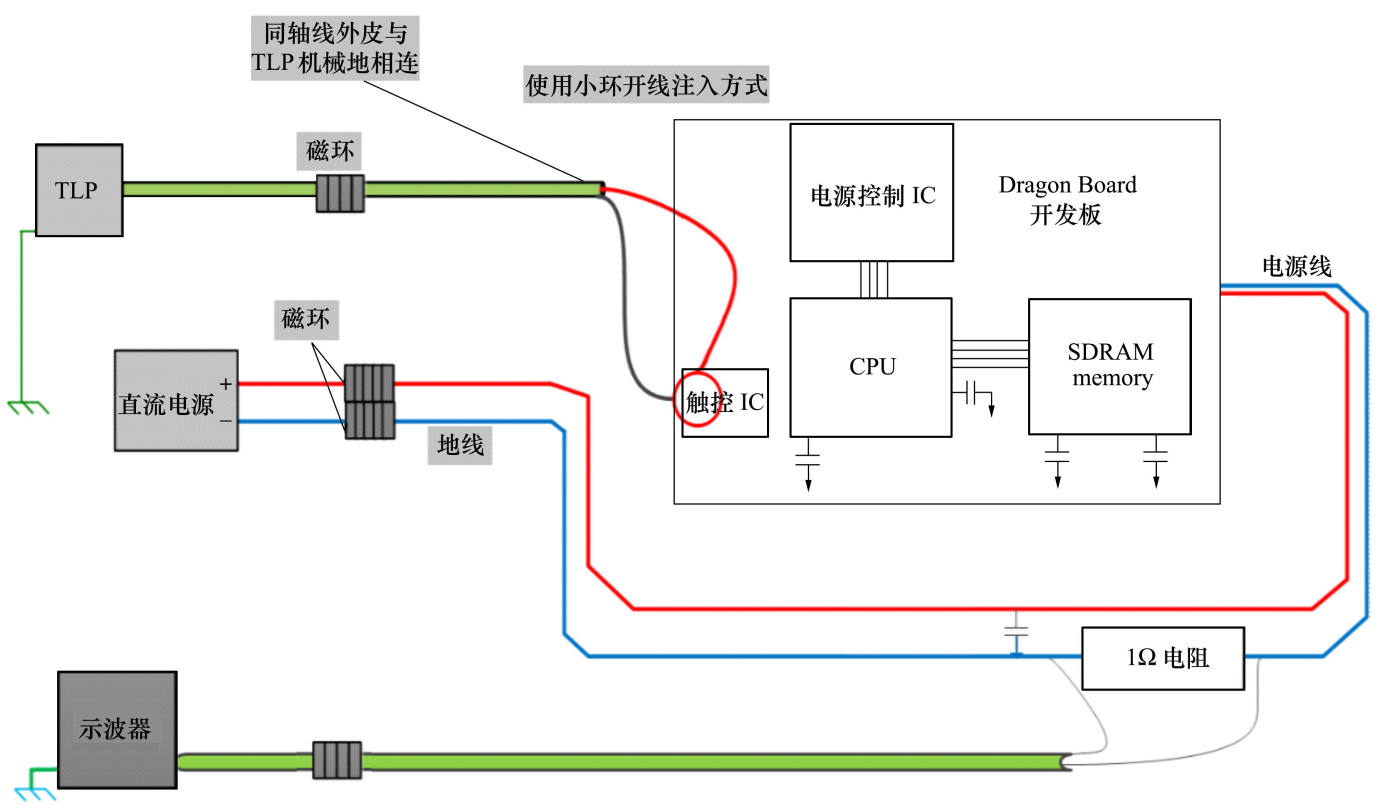


图 2 对比了 DUT 正常运行与 TLP 注人前后电 源回线中直流电流的变化。TLP 注人瞬间开发板指 示灯熄灭, 手动复位后, DUT 能够恢复正常运行。 此软件故障为 $\mathrm{f}$ 类故障。为方便观察, 两次监测的 结果在显示时稍稍错开。从图中可以看出, TLP 注 人后电流增加 $75 \%$, 同时电流波中的周期性脉动消 失。在本实验中, 监测电源回线中的直流电流可以 作为软件故障的监测手段。

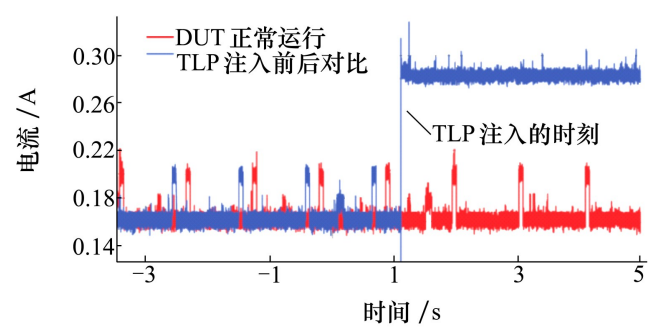

图 2 DUT 正常运行与 TLP 注人前后 2 种 场景下电源回线中的直流电流

此外,也可以使用 I-prober 来监测直流电流。 其实现原理是通过监测电源线周围的磁场来监测线 上的电流值。I-prober 不止可以用于监测电源线的 场合, 也可以用于监测 PCB 中走线上的电流。由于 两种监测手段原理一致,不再赘述。

\section{2 热成像法}

对于一些软件故障, 发生前后会伴随 PCB 上温 度的变化。因此, 考虑使用热成像法作为软件故障 的监测手段之一。

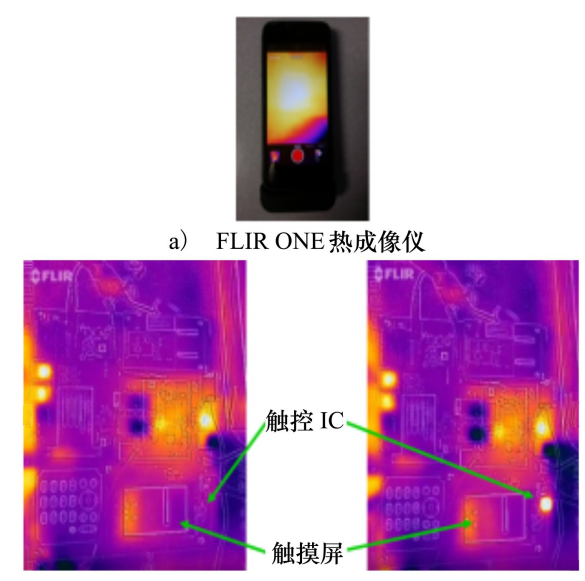

b) TLP 注入前后, 对 DUT 进行成像的对比

图 3 FLIR ONE 热成像仪及其在实验中的应用
本例将热成像法应用于监测高通公司的 Dragon Board 开发板。该开发板使用 APQ8060 CPU, 搭载 Android 系统。TLP 充电电压为 $3 \mathrm{kV}$, 使用小环天线 进行注人。实验中使用了 FLIR ONE 热成像仪。实 验时,FLIR ONE 需要与 1 台 iPhone 配合使用,其灵 敏度为 $0.1{ }^{\circ} \mathrm{C}$ 。

图 3b) 对比了 DUT 正常运行与 TLP 注人后的 热成像图。TLP 注人后触屏不能正常工作, 但系统 软件正常运行。重启后该故障消失。此软件故障为 $\mathrm{b}$ 类故障。当软件故障出现时, 在触控 IC 处出现很 明显的亮点, 说明该处存在明显的温升。在本实验 中, 监测 DUT 的热成像可以作为软件故障的监测 手段。

\section{3 监测直流电压法}

对于一些软件故障, 发生前后会伴随 PDN 中电 压的明显波动。因此, 考虑使用测量直流电压的方 法作为软件故障的监测手段之一。

本例将测量直流电压的方法应用于监测 Beagle Bone Black 开发板出现的软件故障。TLP 充电电压 为 $3.5 \mathrm{kV}$, 同样使用小环天线的注人方式。

实验配置如图 4 所示。

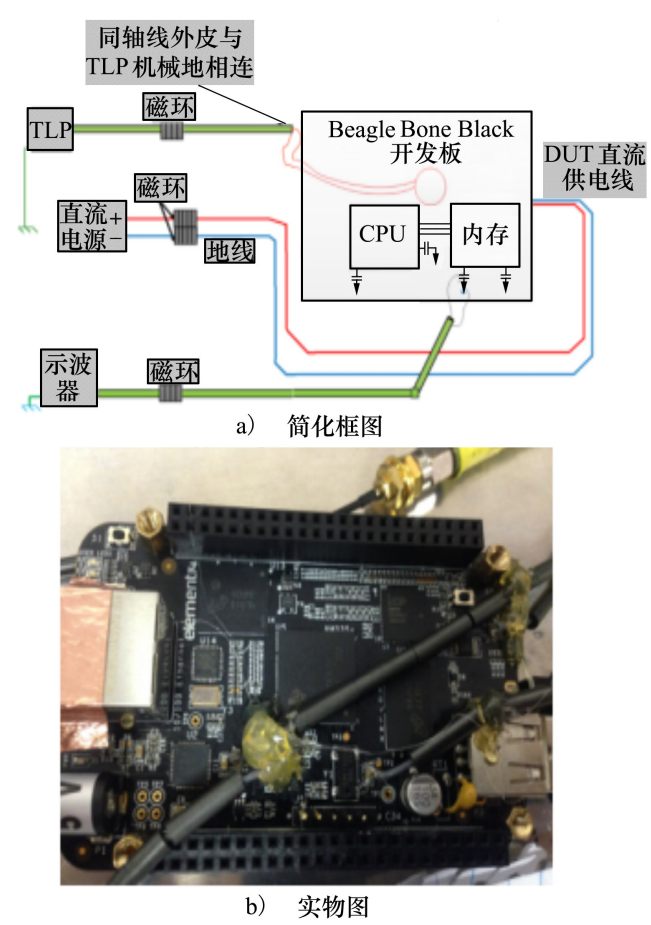

图 4 Beagle Bone Black 开发板上监测直流 电压的实验设置 
首先, 需要配合原理图在 PCB 上量取一些去耦 电容的端电压, 寻找不同的 PDN。其后, 在不同 PDN 的去耦电容两端焊接引线, 使用多通道示波器 同时测量 TLP 注人前后各 PDN 电压的变化。为了 降低电源适配器开关噪声潜在的影响, 可在电源线 上并接电容。同时, 为了降低潜在的共模噪声, 在示 波器通道引线上串接磁环。通过将注人环天线停放 在 DUT 不同的位置, 得到不同的软件故障, 同时监 测得到对应时刻各 PDN 上的电压变化。与前例相 同, 本例依然使用了小环天线进行注人, 即场耦合的 干扰方式。

图 5 和表 1 对比了在 Beagle Bone Black 开发板 上监测的 4 个直流电压。TLP 注人后, 开发板系统 崩溃并随后复位。该类软件故障为 $\mathrm{c}$ 类故障。在本 实验中, $\mathrm{PCB}$ 上各 $\mathrm{PDN}$ 上的电压变化可以作为软件 故障的监测手段。

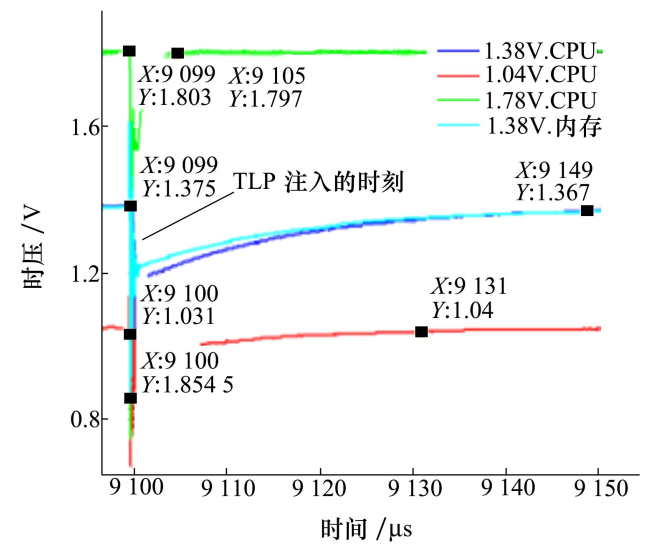

图 5 Beagle Bone Black 开发板上监测的直流电压

\section{表 1 Beagle Bone Black 开发板上监测的直流电压}

\begin{tabular}{cccc}
\hline $\begin{array}{c}\text { 监测的 } \\
\text { 电压点 }\end{array}$ & $\begin{array}{c}\text { 电压波形下 } \\
\text { 降沿时间 } / \mathrm{ns}\end{array}$ & $\begin{array}{c}\text { 电压最 } \\
\text { 低值 } / \mathrm{V}\end{array}$ & $\begin{array}{c}\text { 电压恢 } \\
\text { 复时间 } / \mu \mathrm{s}\end{array}$ \\
\hline $1.38 \mathrm{~V}(\mathrm{CPU})$ & $<5$ & 0.9 & 50 \\
$1.04 \mathrm{~V}(\mathrm{CPU})$ & $<5$ & 0.8 & 30 \\
$1.78 \mathrm{~V}(\mathrm{CPU})$ & $<5$ & 0.7 & 5 \\
$1.38 \mathrm{~V}($ 内存 $)$ & $<5$ & 0.8 & 50 \\
\hline
\end{tabular}

\section{4 频谱观察法}

受 TLP 影响后,一些 DUT 产生软件故障的同 时, 产生的异常信号包含某些高频分量。由于高频 分量会向周围空间进行辐射, 因此考虑使用另一个
小环天线采集 PCB 周围空间的高频分量, 并使用频 率分析仪观察 TLP 注人瞬间, 软件故障发生前后的 频谱变化。

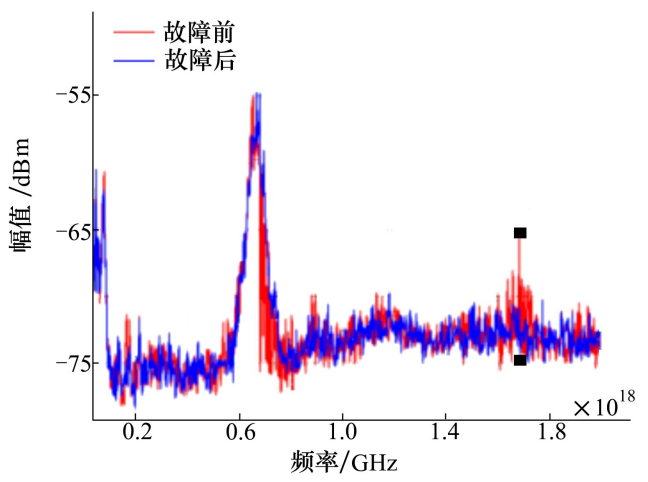

图 6 通过频谱分析仪对比 TLP 注人前后频谱的变化

本例将观察频谱变化的方法应用于监测 1 台智 能手机 (型号:Xperia Arc) 出现的软件故障。TLP 通 过电阻耦合的方式直接注人 DUT 的地平面。TLP 注人后, 整个系统崩溃, 但手机供电正常。这是一个 $\mathrm{c}$ 类软件故障。监测环天线位于 $\mathrm{CPU}$ 上方, 图 6 给 出了 TLP 注人前后的频谱变化, 可以看出在 $1.6 \sim$ $1.7 \mathrm{GHz}$ 之间, DUT 系统崩溃后频谱幅度明显下降。 在本实验中,频谱观察法可以作为软件故障的监测 手段。

\section{5 快速短时傅里叶变换分析法}

由于使用频谱分析仪只能观测到毫秒级到秒级 别的变化, 需要功能更为强大的分析工具来捕捉微 秒甚至纳秒级的变化, 为此笔者提出了快速短时傅 里叶变换分析法,并搭建了快速短时傅里叶变换分 析平台。

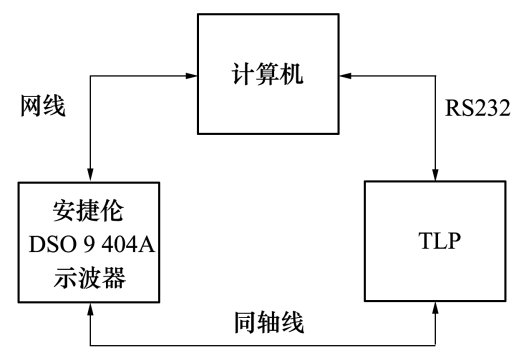

图 7 快速短时傅里叶变换分析平台架构

图 7 展示了笔者所开发的快速短时傅里叶变换 分析平台。计算机与 TLP 通过串口进行通信, 以发 送脉冲波形、脉冲周期、脉冲触发等指令。PC 与示 
波器 (型号: 安捷伦 DSO 9404A) 通过网口进行通 信, 以控制示波器各通道的采样频率、采样点数、触 发方式等内容。TLP 为示波器提供外部触发。计算 机操控平台为用 MATLAB GUI 开发的在线分析 软件。

快速短时傅里叶变换分析平台功能十分强大。 它可以分析纳秒到毫秒级的时域信号, 通过调节参 数, 可以同时在时域和频域都得到较高的分辨率。

本例将观察频谱变化的方法应用于监测 Dragon Board 开发板出现的软件故障。TLP 通过小环天线 的方式进行注人，注人环天线位于 SD 卡槽的上方。 监测环天线位于 CPU 上方。DUT 的工作状态为播 放 SD 卡中的视频文件。TLP 注人后, 视频停止播 放,并自动返回文件选择界面。系统软件运行正常。 这是一个 $\mathrm{a}$ 类软件故障。图 8 给出了 TLP 注人前后 的频谱变化, 可以看出在方框 1 内, 一些频率分量显 著减弱。在本实验中, 快速短时傅里叶变换法可以 作为软件故障的监测手段。
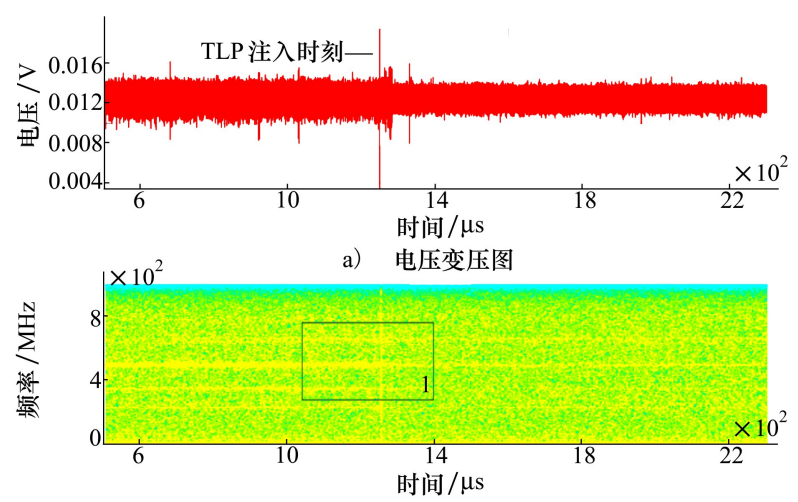

b）频率变压图

图 8 使用快速短时傅里叶变换法监测 TLP 注人前后频谱的变化

\section{6 声音辨别法}

有时高频范围的微小变化可能难以被测量仪器 监测到, 但可能通过将高频信号转换到听觉范围内, 用耳听的方法来识别。这是一种软件故障评估的主 观方法。
这里通过一个在 Dragon Board 开发板上发现的 软件故障来演示声音辨别法。实验中, 注人环天线 位于 $\mathrm{WiFi}$ /蓝牙 $\mathrm{IC}$ 上方, 将开发板的工作状态设置 为通过 WiFi 进行数据传输。 TLP 注人后, 数据传输 停止, 但系统依然正常工作, 因此这是一个 $\mathrm{b}$ 类软件 故障。图 9 是监测环天线采集到的时域信号。单从 图上看, 除了 TLP 注人信号和后面的反射信号, 难 以辨别 TLP 注人前后的变化。但通过将该高频信 号转换到听觉范围内, 可以辨别出 TLP 注人前后开 发板系统的变化。该变化可以与 $\mathrm{WiFi}$ 模块的受扰 现象相对应。在本实验中, 声音辨别法可以作为软 件故障的监测手段。

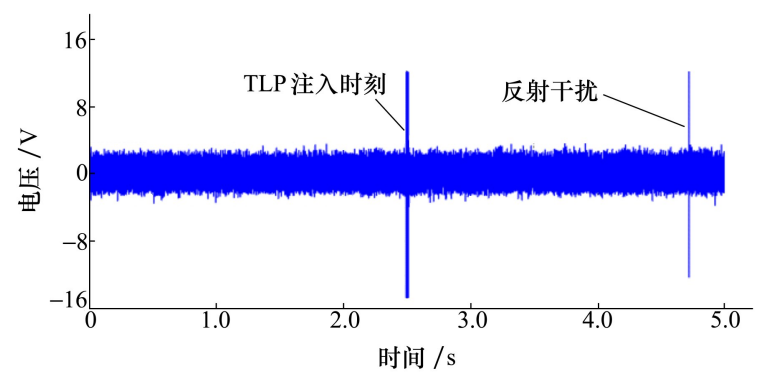

图 9 监测环天线采集到的时域信号

\section{3 监测手段的适用性}

上面一共提出了 6 种方法。本节将介绍每种方 法的适用场景。从一定数量的实验中, 可以初步得 到软件故障类别与适用的探测方法之间的关系。总 体来说, 对于一个典型的 DUT, 并不是前面提出的 每种监测手段都适用于软件故障的预测, 甚至对于 同一个 DUT 的不同工作状态而言同样不成立。

表 2 总结了每种方法的适用场景。表 2 基于笔 者现有的实验。软件故障类别和可能有效的预测方 法需要后续更为深人的分析研究。

到目前为止, 从大量实验中可以总结得到, 快速 短时傅里叶变换法是最强大的软件故障预测方法。 
表 2 监测手段场景适用性

\begin{tabular}{|c|c|c|}
\hline 监测手段 & 适用场景 & 受限条件 \\
\hline 直流电流监测法-使用 $1 \Omega$ 电阻 & $\begin{array}{l}\text { 电流变化集中在低频, 且幅度明显。如 } \\
\text { 主要应用程序崩溃、系统闭锁等 }\end{array}$ & $\begin{array}{l}\text { 适用频率范围仅为 } \mathrm{kHz} \text { 级; 不能探测到是系 } \\
\text { 统的哪些模块导致电流变化 }\end{array}$ \\
\hline 直流电流监测法-使用 I-prober & 可以监测 $\mathrm{PCB}$ 走线上 $\mathrm{mA}$ 级别的电流 & 适用频率范围为 $5 \mathrm{MHz}$ 以下 \\
\hline 热成像法 & $\begin{array}{l}\text { 可以快速定位板上电流变化较大的 } \\
\text { 区域 }\end{array}$ & $\begin{array}{l}\text { 无法提供电流变化的时域和频域信息; 无法 } \\
\text { 探测到 } 0.1 \mathrm{~ms} \text { 以内的电流变化 }\end{array}$ \\
\hline 直流电压监测法 & 可以探测到 $\mathrm{mV}$ 级别的电压变化 & $\begin{array}{l}\text { 适用频率范围为数百 } \mathrm{kHz} \text { 到数 } \mathrm{MHz} \text {; 需要焊 } \\
\text { 接引线 }\end{array}$ \\
\hline 频谱分析法-使用频谱分析仪 & 监测 $\mathrm{ms}$ 或 $\mathrm{s}$ 级的变化 & $\begin{array}{l}\text { 适用于探测 } \mathrm{ms} \sim \mathrm{s} \text { 级的变化; 需要人员具备 } \\
\text { 操控经验以发现 TLP 注人前后的频谱变化 }\end{array}$ \\
\hline 频谱分析法-短时傅里叶变换 & 可以探测低至 $n s$ 和 $\mu \mathrm{s}$ 级的变化 & $\begin{array}{l}\text { 从示波器下载时域数据后在计算机进行短 } \\
\text { 时傅里叶变换处理十分费时 }\end{array}$ \\
\hline 声音辨别法 & $\begin{array}{l}\text { 可能探测到监测仪器无法分辨的高频 } \\
\text { 分量变化 }\end{array}$ & 分辨结果受限于主观因素判断 \\
\hline
\end{tabular}

\section{4 软件故障的预测方法}

实验目的是探求软件故障的预测方法。如果针 对某一类软件故障能够找到对应的预测方法, 那么 就有可能在产品设计阶段解决该类问题。

本节将演示什么样的软件故障监测手段可能成 为预测方法。实验中使用快速短时傅里叶变换法作 为监测手段。DUT 是 Dragon Board 开发板。注人环 天线位于 $\mathrm{WiFi} /$ 蓝牙 $\mathrm{IC}$ 上方, 监测环天线位于 SDRAM 上方。DUT 的工作状态为通过蓝牙传输文 件。注人 TLP 后, 蓝牙功能受扰, 产生软件故障, 对 应现象为文件停止传输 ( $\mathrm{b}$ 类故障)。随后人为重启 开发板, 并使其处于待机状态 (不使用蓝牙功能), 使用与前面实验相同的注人环天线和监测环天线， 保持注人位置和监测位置不变并注人 TLP。图 10 显示 TLP 注人后软件故障发生前后快速短时傅里 叶变换的结果。图 11 显示 DUT 处于待机状态下注 人 TLP 前后短时傅里叶变换的结果。

从本例中可以看出, 无论蓝牙模块工作与否, 快 速短时傅里叶变换的方法都能够探测到 TLP 注人 前后的变化。因此, 快速短时傅里叶变换的方法可 以用作预测软件故障的一个候选方法。
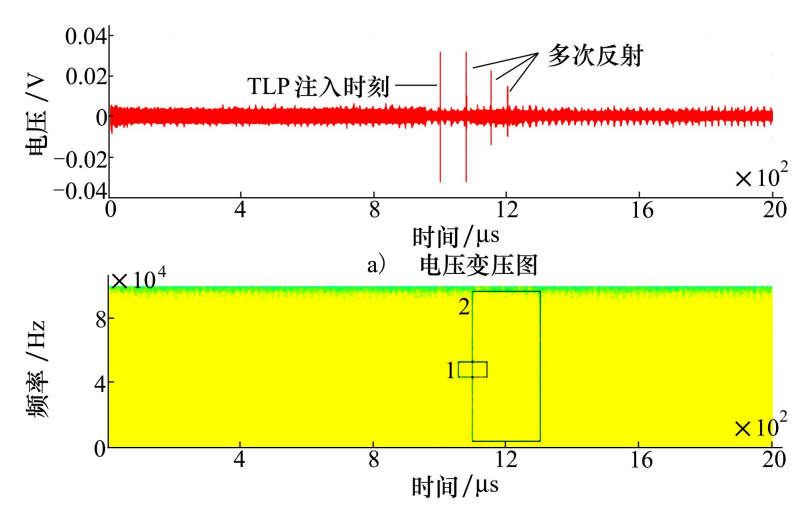

b) 频率变压图

图 10 注人 TLP 后软件故障发生前后短时傅里叶变换的结果
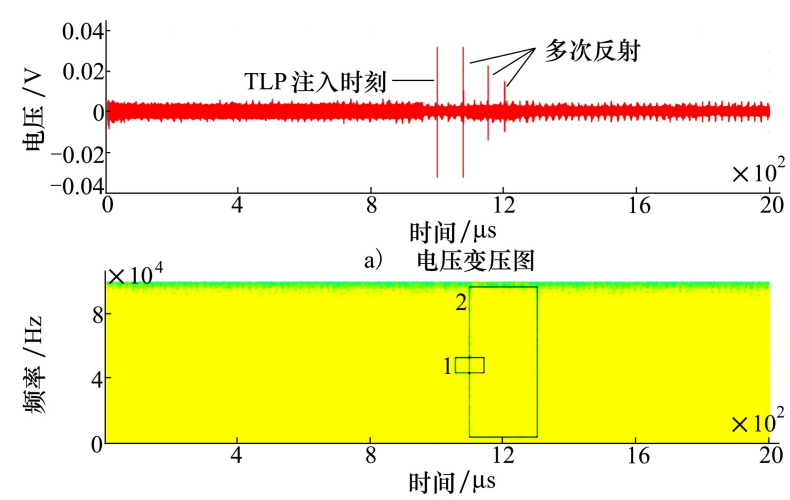

b) 频率变压图

图 11 待机状态下注人 TLP 前后短时傅里叶变换的结果 


\section{5 结 论}

根据实验现象对发现的嵌人式软件故障进行了 分类,介绍了 6 种软件故障的监测手段,并讨论了每 种方法的适用条件和使用限制。需要强调的是, 针
对不同类别的软件故障、同类别软件故障下不同的 DUT 甚至同一个 DUT 的不同的工作状态, 监测手段 都可能不同。所以,需要针对具体产品的具体工作 状态进行单独实验和分析。后续,笔者着眼于发现 更多的软件故障监测手段,并试图从中得到更多的 软件故障预测方法。

\section{参考文献:}

[1] Mark I Montrose, Edward M Nakauchi. Testing for EMC Compliance: Approaches and Techniques[M]. John Wiley \& Sons, 2004: 93-94

[2] Iec61000-4-2, Testing and Measurement Techniques-Electrostatic Discharge Immunity Test [S]. Tc 77/Sc 77B-High Frequency Phenomena, 2008-12-09

[3] Charvaka Duvvury, Harald Gossner. Soft Failure Mechanisms and PCB Design Measures[M]. Wiley-IEEE Press, 2015 : 424

[4] Thomson N A, Xiu Y, Rosenbaum E. Soft-Failures Induced by System-Level ESD[J]. IEEE Trans on Device and Materials Reliability, 2017, 17(1): 90-98

[5] Vora S, Jiang R, Vasudevan S, et al. Application Level Investigation of System-Level Esd-Induced Soft Failures [ C] // 2016 38th IEEE Electrical Overstress/Electrostatic Discharge Symposium, 2016: 1-10

[6] Jin J H, Jeon C P, Kim J H, et al. The Reproducibility Improving Method of Systemlevel ESD Test Through Operating Program Workload Analysis[C] //URSI Asia-Pacific Radio Science Conference, 2016: 921-924

[7] Schwingshackl T, Orr B, Willemen J, et al. Powered System-Level Conductive TLP Probing Method for ESD/EMI Hard Fail and Soft Fail Threshold Evaluation[C] //2013 35th IEEE Overstress/Electrostatic Discharge Symposium, 2013: 1-8

[8] Lim J, Lee J, Seol B, et al. The Evaluation Method for ESD Immunity of Components in Terms of Software Error[C] // EMC Europe, 2011: 468-471

[9] Yang S, Orr B, Guo Y, et al. Measurement Techniques to Predict the Soft Failure Susceptibility of an IC without the Aid of a Complete Software Stack [C] //2016 IEEE International Symposium on Electromagnetic Compatibility, 2016: 41-45

[10] Antong R, Low D, Pommerenke D, et al. Prediction of Electrostatic Discharge( ESD) Software Error on Two-Way Radio Using ESD Simulation in CST and ESD Immunity Scanning Technique[C] // 2014 36th IEEE Electronics Manufacturing Technology Symposium, 2014: 1-10

[11] Lee J, Lim J, Jo C, et al. A Study of a Measurement and Simulation Method on ESD Noise Causing Soft-Errors by Disturbing Signals[C] //2011 33rd IEEE Electrical Overstress/Electrostatic Discharge Symposium, 2011: 1-5

[12] Lee J, Lim J, Seol B, et al. A Novel Method for ESD Software Error Analysis on Integrated Circuits Using a Tem Cell [C] // 2012 34th IEEE Electrical Overstress/Electrostatic Discharge Symposium, 2012: 1-6

[13] Lim Jaedeok, Lee Jongsung, Seol B, et al. The Quantitative Approach of EM-Field to Correlate Components and Products in Terms of Esd Software Error[C] // 2013 International Symposium on Electromagnetic Compatibility, 2013: 916-919 


\title{
Equivalent Experiments Based Prediction Methods for Embedded Software Error due to Electrostatic Discharge
}

\author{
Qin Haichao
}

(School of Electronic and Information Engineering, Beihang University, Beijing 100191, China)

\begin{abstract}
Electrostatic discharge (ESD) related embedded software error is a critical issue for electronic products and is hard to discover in the early stage of product development. This paper categorizes different embedded software errors at first, and then presents several detection methods to find precursors of these software errors. By discussing the methodology, the applicability of each method is given, in terms of application scenarios and limitations.
\end{abstract}

Keywords : embedded software testing; electro-static discharge; design of experiments; WiFi 